\title{
The Largest Reported Dissecting Aneurysm of Ascending Aorta Following Aortic Valve Replacement Accompanied by Superior Vena Cava Syndrome
}

\author{
Feridoun Sabzi $^{1}$, Donya Khosravi ${ }^{2}$, Maryamosadat Hosseini ${ }^{2}$, Reza Faraji ${ }^{3}$
}

\begin{abstract}
BACKGROUND: Ascending aortic dissection (AAD) is a rare and serious complication of aortic valve replacement. Multiple risk factors such as connective tissue disease, aortic wall thinning, aortic diameter, calcification of wall, structural features of aortic wall and associated diseases have been considered as a predisposing factor for the occurrences of $A A D$. Preoperative recognition of these variables with proper intra intra operative logic judgment may decrease tehe incidence of this complication.

CASE DETAILS: We herein present a huge ascending aorta with dissecting aneurysm (AAD) with a largeintra-operative diameter $(15 \mathrm{~cm})$ that has not been recorded in the medical literature so far. He presented with dyspnea, chest pain and amazing symptom of superior vena cava syndrome. The patient underwent open heart surgery with resection of ascending aorta aneurysm with classic Bentall operation. The post-operative period was associated with uneventful course and the patient was discharged with good condition on $12^{\text {th }}$ post-operative day.

CONCLUSION: A six months' follow-up revealed abolishment of chest pain and superior vena cava (SVC) syndrome and good prosthetic composite graft function with no recurrence of pseudo aneurysm or dissection.
\end{abstract}

KEYWORDS: aneurysm, ascending aorta, superior vena cava syndrome

DOI: http://dx.doi.org/10.4314/ejhs.v25i3.14

\section{INTRODUCTION}

Aortic dissection is not a rare event in general population but is a very rare and serious complication in post aortic valve replacement. However, the incidence of aortic dissection (type A) varies between 3.5 to 9 per 100,000 persons in general population, but this prevalence is much higher and reaches between 0.6 to $1 \%$ in post AVR surgery. Although AVR itself is considered as an independent risk factor for the development of subsequent dissection, the preoperative and the patient's risk factors also determinate occurrences of post-operative dissection $(1,2)$. These risk factors include aortic insufficiency, systemic hypertension, male gender, thinned or fragile aortic wall and ascending aortic dilatation (3). In this article, we present a successful surgical treatment of a giant dissecting aneurysm of ascending aorta late after aortic valve replacement with large diameter $(15 \mathrm{~cm})$. The largest reported dissecting aneurism in post AVR surgery $(9 \mathrm{~cm})$ related to Fukui study was smaller than our case (4). However, SVC syndrome was reported in post AVR surgery but two unique features of ours case related to size of dissecting aneurism and SVC syndrome that rarely has been reported in medical literature. The most common causes of superior venacava syndrome are mediastinal tumors that by

\footnotetext{
${ }^{1}$ Preventive Cardiovascular Research Centre Kermanshah, Kermanshah University of Medical Sciences, Kermanshah, Iran

${ }^{2}$ Department of Gynecology, School of Medicine, Shahid Beheshti University of Medical Sciences, Preventive Gynecology Research Center, Tehran, Iran

${ }^{3}$ Yazd Cardiovascular Research Center, Shahid Sadoughi University of Medical Sciences, Yazd, Iran

Corresponding Author: Reza Faraji, Email:r.faraji61 @ g mail.com
} 
external compression or intra luminal invasion of the cava leadd to SVC syndrome. Among benign causes, the dissected pseudoaneurysm of the ascending aorta in post AVR period is exceedingly rare.

\section{CASE REPORT}

A 67-year-old man with cough, chest pain and dyspnea and intermittent symptom of superior vena cava syndrome (SVCS) as upper thorax plethora, dilated jugular veins, dark blue change of neck and severe headache from 4 weeks earlier was referred to our center from another hospital. His aforementioned symptoms were aggravated by bending forward, straining and cough. As his dyspnea worsened, he was referred for transe thoracic echocardiography examination after a widening of mediastinum was seen on chest radiography. The patient's history showed prosthetic aortic valve replacement with carbomedic (23 number of sorin group) that has been performed 10 years earlier. In the previous operation, risk factors such as coarectation, bicuspid aortic valve, aortic wall, calcification or thin aortic wall were not reported. However, patient's native valve and its ring was calcified, and ascending aorta had a diameter of $41 \mathrm{~mm}$. Physical examination revealed no murmur along the precordium.The patient was dyspnoeic with chest pain. His blood pressure was normal and the oxygen saturation without oxygen supply was $92 \%$. ECG showed atrial fibrillation with rapid ventricular response.

Physical examination on admission showed mild cyanosis and plethora of head and neck and distended jugular veins. SVCS was clinically diagnosed. Laboratory data included an INR of 3 and $\mathrm{Ht}: 35 \%$. Other components of blood count and coagulation time were within physiologic values. Cardiac enzymes were negative. LDH was $620 \mathrm{IU} / \mathrm{L}$. The other biochemical tests were normal. Arterial blood gases showed mild respiratory acidosis (PH: 7.33) due to elevated $\mathrm{PCO}_{2}: 48 \mathrm{mmHg}$. Chest X-ray revealed a widened mediastinum. The contrast CT angiography of the chest was remarkable, revealing a large aneurysm of the ascending aorta with a maximum diameter of $15 \mathrm{~cm}$ compressing the superior vena cava. However, intimal flap was not detected in this modality (Figure 1). Venography revealed compression of SVC by bulging of aneurysm
(Figure 2). Transthoracic echocardiography (TEE) showed normal functional prosthetic aortic valve and ascending aortic aneurysm involving sinuses of valsalva. However, dissection flap was not reported. The maximum size of aneurysm by TEE was $7 \mathrm{~cm}$. There was a discrepancy between reported size of aneurysm in TEE and C-T angiography and intra operative inspection. The gross size of aneurysm was much larger than the one reported in TEE (Figure 3). Aneurysm's transverse diameter was $15 \mathrm{~cm}$ with C-T angiography and gross examination. Right coronary arteries were found normal by angiography, but LAD and LCX were not detected by angiography (Figure 4). The patient underwent redo open heart surgery. The CPB was instituted by femoral artery, and right atrial cannulation and midline sternotomy were performed. A huge ascending aorta aneurysm attached to SVC by inflammatory reaction and subsequently leading to its narrowing was detected intraoperatively (Figure 5). Following ascending aorta clamping, aortotomy was performed and direct cold cardioplegia was delivered to both coronaries ostium (Figure 6). Aneurysm was transected just above the right coronary artery ostium. Both right and left coronary ostium bottoms were prepared and released from the surrounding structures. Due to aneurysm of valsalva sinuses and despite normal prosthetic aortic valve function, modified Bentall operation was not performed. The supra annular space as valsalva sinus had abnormal tissue consistency, and was not appropriate for modified Bentall surgery. The prosthetic valve was removed and classic Bentall procedure was performed with no: 25 carbomedic valved conduit composite graft of sorine group. The operation was completed with anastomosis of Dacron tube to proximal normal ascending aorta. Postoperative course was uneventful. The patient was discharged on the $12^{\text {th }}$ day of the postoperative period in well condition. Our patient had two unique feature i.e. huge diameter of aneurism $(15 \mathrm{~cm})$ and superior vena cava syndrome. 


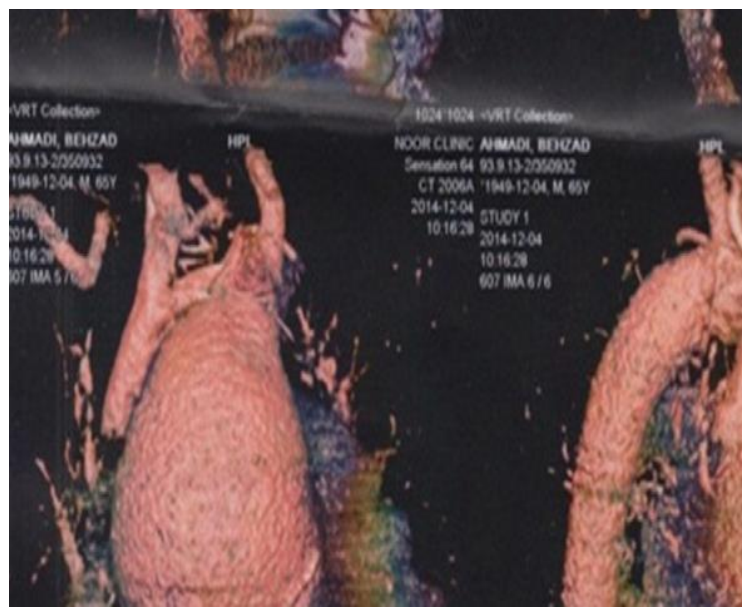

Figure 1: C-T angiography shows huge ascending aorta aneurysm

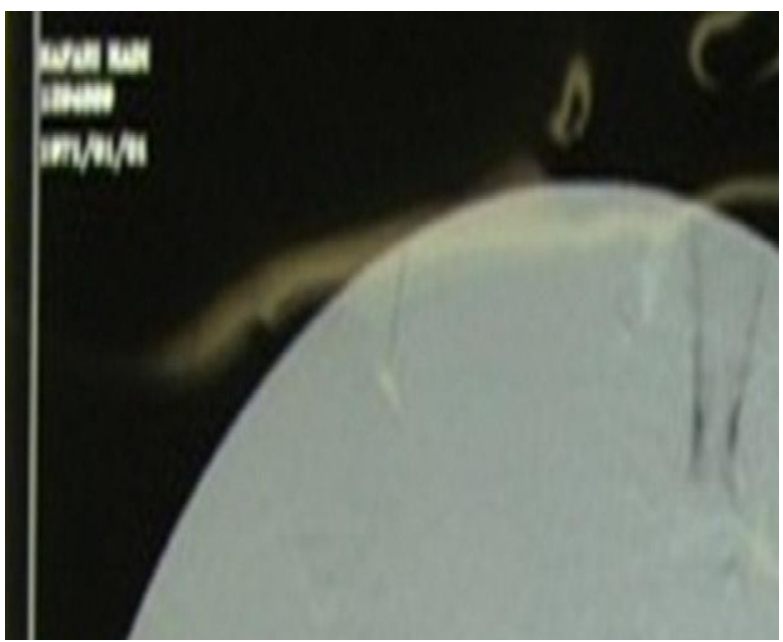

Figure 2: Shows pressure effect of dissecting aneurism on superior vena cava

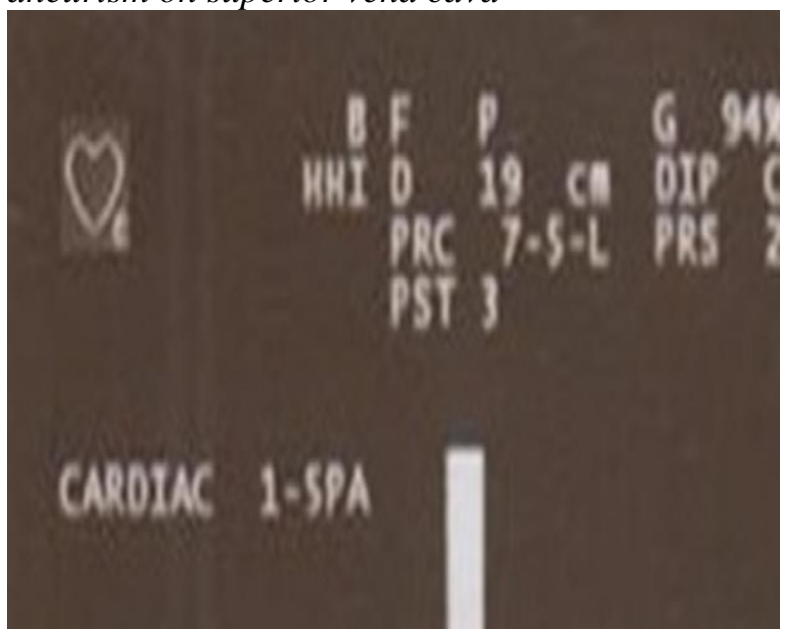

Figure 3: Transe thoracic echocardiography shows large aneurysm

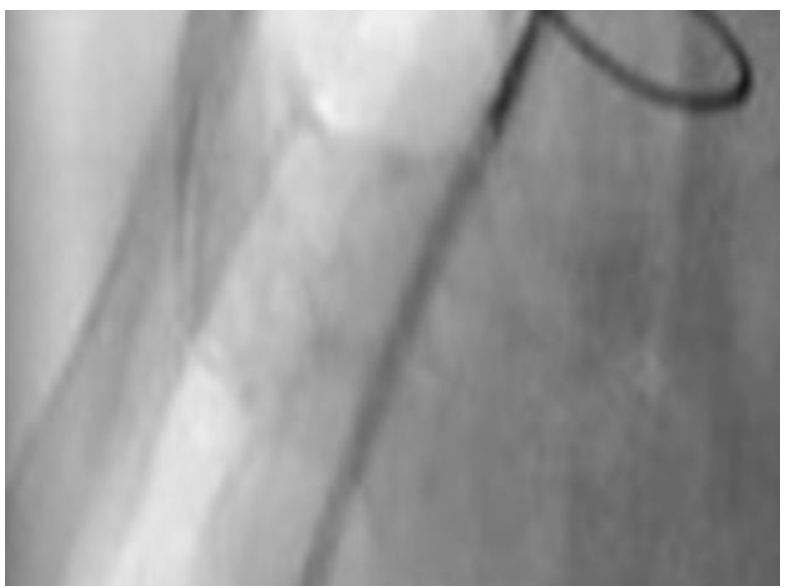

Figure 4: Angiography shows huge ascending dissecting aneurism with prosthetic valve

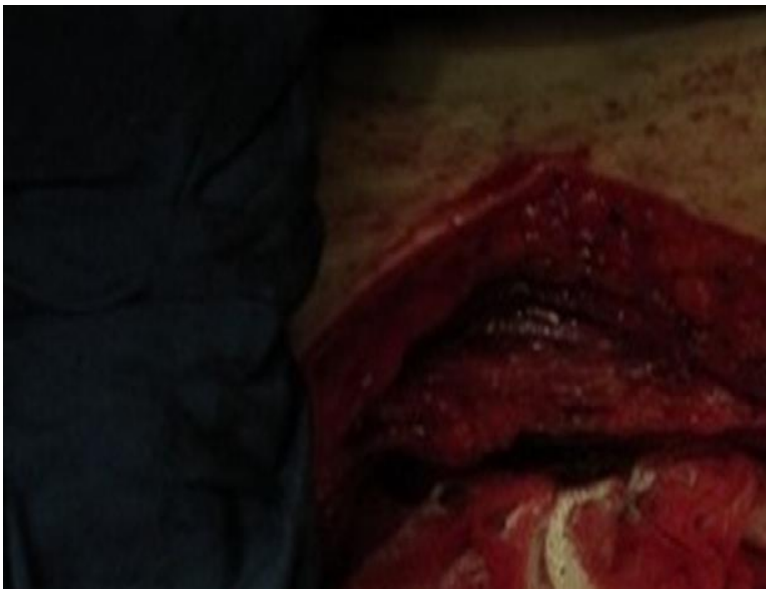

Figure 5: Shows huge size of ascending aorta in intra operative view

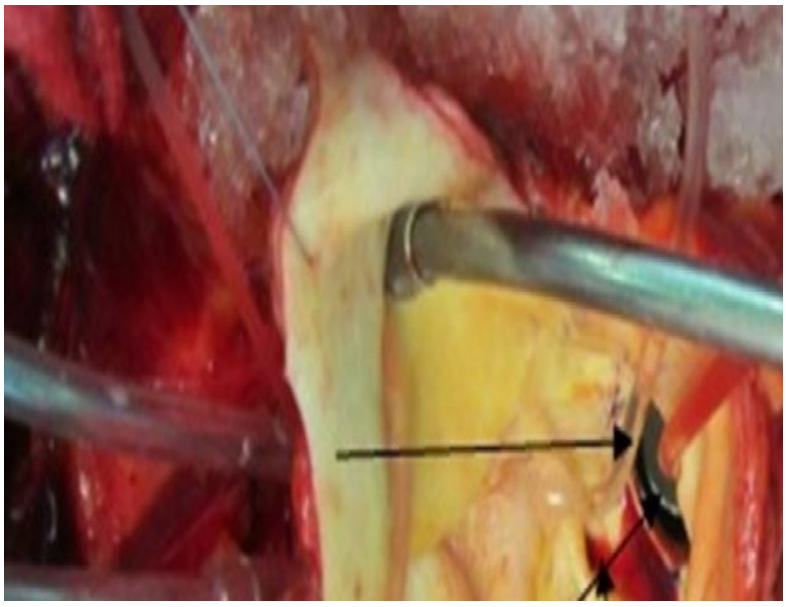

Figure 6: Shows left main coronary ostium (vertical arrow), cardioplegin right angel cannula (transverse arrow) and intimal flap (curve arrow) immediately above the coronary ostium and prosthetic aortic valve (oblique arrow) 


\section{DISCUSSION}

Factors predicting aortic root complication after AVR could be classified as preoperative,intra operative and post-operative variables. Preoperative variables include, hypertension, aortic root dilatation, bicuspid aortic valve, connective tissue disorder (Marfan syndrome, vascular Ehlers-Danlos syndrome, Turner syndrome, and Loeys-Dietz syndrome) (5). Any concomitant procedure that needs accomplishment of AVR such as aortic root canuula for cardioplegin, aortotomy site decalcification of aortic ring or aorticvalves, partialor or complete aortic clamping site, accidental trauma to aortic wall by electrocautery, and use of suture with large needle (5-7). Post-operative factors include hypertension and infection of suture lines. However, these complications are rare which potentially have serious consequence. The most important factor for predicting pos-toperative dissection after AVR is aortic root dilatation. When the aorta has a markedly enlarged diameter $(>50 \mathrm{~mm})$ at initial AVR, combined ascending aortic replacement is a preferable surgical procedure. However, it is an issue of controversy whether a mildly dilated ascending aorta $(40-50 \mathrm{~mm})$ should be replaced. In one, study, aortic regurgitation, systemic hypertension, male sex and a thinned or fragile aorta with mild dilation ( $>45 \mathrm{~mm}$ ) at initial AVR have been considered as risk factors for late aortic complications. It seems that management of dilated aorta in the AVR candidate patients depended on coexisting risk factors observed in the pre and intra operative periods $(8-10)$. There is no specific time frame for occurrences of dissection pos-toperatively. Time interval from initial AVR to dissection ranges from one to18 years. Post-operative dissection occurs in all AVR surgery that aorta is handled during initial surgery. The dissection's flap can begin at multiple location, such as aortic cannulation site, cardioplegia cannula site, aortic cross clamping site, aortotomy site and in severe atherosclerosis of aortic wall. It is vital to carefully close the aortotomy site with small needle and sutures close together that have appropriate distance from the aortotomy at the initial surgery. In our case, the intimal tear was found immediately above the left main coronary artery ostium in sinotubular junction; however, the entry site had enough distance from the previous aortotomy line. It seems that direct cardioplegin perfusion to left coronary ostiummay caused small intimal tear that extended upward to posterior aortic wall in this patient with fragile aortic wall. There is not logical consensus about management of dilated aorta in initial AVR. Some surgeons consider an aortic diameter limit of $44 \mathrm{~mm}$ as maximum diameter that could be treated by aortoplasty or replacement in initial AVR. Other surgeon believe that aortic diameter between 45 to $50 \mathrm{~mm}$ is an issue of debate (11). Most cardiac surgeons refrain to perform replacement or aortoplasty in patients with concomitant risk factors such as low ejection fraction, elderly patients' renal dysfunction or history of COPD or other organ dysfunctions. Prolonged cardiopulmonary bypass may lead to further cardiac dysfunction, cerebral complication, respiratory dysfunction, acute renal failure, which can increase post-operative ICU stay and increase morbidity and mortality (12). To avoid a prolonged CPB time, some surgeons use external aortoplasty or reinforcement with Dacron tube. More studies are necessary to conclude whether both of these conservative surgeries could be considered as an alternative to aortic replacement in high risk patients to prevent future dissection. All the aforementioned surgical approaches may be associated with SVCS. If SVCS occurred following the pseudo aneurysm of aorta, it usually results from extrinsic compression of vessel (13). Etiology of SVCS has been changed considerably in recent decades. In McIntire and Sykes's study in a case series of SVCS 60 years ago, $70 \%$ of SVCS were caused by benign etiology of which the most common causes were secondary to syphilitic aneurysms $(55 \%)$ and $15 \%$ were secondary to other mediastinal inflammation such as tuberculosis, sarcoidosis or mediastinal fibrosis, whereas $30 \%$ were caused by malignant tumors (14). In recent decades, the common etiology of SVCS has reversed from infection causes to malignancy (15). In a recent study by Baker, malignant and benign diseases accounted for $90 \%$ and $10 \%$ of cases respectively. Today, SVCS is almost always observed as a complication caused by lung malignancy. Due to low incidence, benign causes of SVCS are often missed in the differential diagnosis (16). However, post-cardiac surgery cause of SVCS is easily detected by recent development of imaging modality and 
differentiated from rare benign mediastinal mass, inflammatory pseudo tumor, autoimmune or granulomatous processes. Dissection of a post AVR ascending aorta producing huge pseudo aneurysm is an exceedingly rare cause of SVCS (17).

\section{REFERENCES}

1. Von Kodolitsch Y, Loose R, Ostermeyer J, Aydin A, Koschyk DH, Haverich A, et al. Proximal aortic dissection late after aortic valve surgery: 119 cases of a distinct clinical entity. Thorac Cardiovasc Surg 2000;48:3426. doi: 10.1055/s-2000-8346.

2. Stanger O, Oberwalder P, Dacar D, Knez I, Rigler B. Late dissection of the ascending aorta after previous cardiac surgery: risk, presentation and outcome. Eur J Cardiothorac Surg 2002;21:453-8. doi: 10.1016/S10107940(01)01144-7.

3. Tsutsumi K, Inoue Y, Hashizume K, Kimura N, Takahashi R. Risk factor analysis for acute type A aortic dissection after aortic valve replacement. Gen Thorac Cardiovasc Surg 2010;58:601-5. doi: 10.1007/s11748-0100658-z.

4. Fukui T, Ro D, Takanashi S. Superior vena cava syndrome secondary to chronic dissecting aortic aneurysm after aortic valve replacement. Interact CardioVasc Thorac Surg 2010;11:193-192. doi: 10.1510/icvts.2010.238022.

5. Pieters FAA, Widdershoven JW, Gerardy AC, Geskes G, Cheriex EC, Wellens HJ. Risk of aortic dissection after aortic valve replacement. Am J Cardiol 1993;72:1043-7.

6. Von Kodolitsch Y, Simic O, Bregenzer T, Dresler C, Haverich A, Nienaber CA. Aortic valve replacement as an independent predisposing factor for late aortic dissection. $Z$ Kardiol 1998;87:604-12.

7. Picchi A, Focardi M, Capannini G, Mondillo S. Aortic dissection after aortic valve replacement-a case report and literature review. Angiology 2003;54:7159.doi:10.1177/000331970305400612

8. Lavall D, Schäfers HJ, Böhm M, Laufs U. Aneurysms of the ascending aorta. Dtsch Arztebl Int 2012;109:227-33. doi: 10.3238/arztebl.2012.0227.
9. Elefteriades JA, Farkas EA. Thoracic aortic aneurysm clinically pertinent controversies and uncertainties. $J \mathrm{Am}$ Coll Cardiol 2010;55:841-57. doi: 10.1016/j.jacc.2009.08.084.

10. Vahanian A, Baumgartner H, Bax J, Butchart E, Dion R, Filippatos G, et al. Guidelines on the management of valvular heart disease: the task force on the management of valvular heart disease of the European society of cardiology. Eur Heart J 2007;28:268-230. doi: http://dx.doi.org/10.1093/eurheartj/ehl428.

11. Yilmaz A, Dessing T, Botta L. Ascending aorta impending rupture: successful surgical management in a patient with type IV EhlersDanlos syndrome. $J$ Cardiovas Med 2010;11:612-610. doi: 10.2459/JCM.0b013e3283314176.

12. Roseborough GS, Williams GM. Marfan and other connective tissue disorders: conservative and surgical considerations. Semin Vasc Surg 2000;13:272-82.

13. Loeys BL, Schwarze U, Holm T, Callewaert BL, Thomas GH, Pannu H, et al. Aneurysm syndromes caused by mutations in the TGF- $\beta$ receptor. N Engl J Med 2006;355:788-98. doi: 10.1056/NEJMoa055695.

14. McIntire FT, Sykes EM.Obstruction of the superior vena cava. Ann Intern Med 1949;30:925-60. doi:10.7326/0003-4819-305-925.

15. Tzemos N, Therrien J, Yip J, Thanassoulis G, Tremblay S, Jamorski MT, et al. Outcomes in adults with bicuspid aortic valves. JAMA 2008;300:1317-25. doi: 10.1001/jama.300.11.1317.

16. Baker GL, Barnes HJ. Superior vena cava syndrome: etiology, diagnosis and treatment. Am J Crit Care 1992;1:54-64.

17. Stajnic M, Canji T, Panić G, Tomić N, Jelkić $\mathrm{N}$, Kovacević B.The superior vena cava syndrome as a manifestation of dissection of the ascending aorta. Med Pregl 2001;54:3802. 